\title{
AVALIAÇÃO DOS ADUBADO COM LODO DE ESTAÇÃO DE TRATAMENTO DE ÁGUA
}

\section{EVALUATE OF ATTRIBUTE OF A LATOSOL FERTILIZED WITH WATER TREATMENT SLUDGE}

\author{
Adriana Paulo de Sousa Oliveira \\ Universidade Federal de Juiz de Fora - UFJF \\ Jonathas Batista Gonçalves Silva \\ Universidade Federal de Juiz de Fora - UFJF \\ Renata de Oliveira Pereira \\ Universidade Federal de Juiz de Fora - UFJF \\ Ana Silvia Pereira Santos \\ Universidade do Estado do Rio de Janeiro - UERJ
}

\begin{abstract}
RESUMO
Objetivou-se neste trabalho avaliar as propriedades químicas e físicas de um latossolo após aplicação do lodo originado nos decantadores de uma estação de tratamento de água. Foram incorporadas ao solo cinco dosagens de lodo em quatro repetições, sendo uma sem aplicação do lodo e as demais doses definidas de acordo com a concentração de nitrogênio presente no lodo, no solo e pela demanda da planta (gramínea). Os atributos químicos e físicos do solo avaliados antes e após a aplicação do lodo inclui a concentração de $\mathrm{Al}^{+3}, \mathrm{pH}$, capacidade de troca de cátions, soma de bases, saturação por alumínio e por bases, condutividade elétrica, matéria orgânica, os nutrientes $\mathrm{P}, \mathrm{K}, \mathrm{Ca}, \mathrm{Mg}, \mathrm{S}$, os metais $\mathrm{Cu}, \mathrm{Mn}, \mathrm{Fe}, \mathrm{Zn}, \mathrm{Cr}, \mathrm{Ni}, \mathrm{Cd}, \mathrm{Pb}$ e a densidade. De acordo com os resultados obtidos, observou-se que o lodo não apresentou características que indiquem o seu uso como corretivo agrícola e adubo e não permitiu determinar a melhor dose agronômica. A concentração elevada de $\mathrm{Mn}$ no lodo limitou a aplicação em 2,5 $\mathrm{mg} \mathrm{kg}^{-1}$. No entanto, o lodo de ETA foi capaz de reduzir em $20 \% 0$ índice de saturação por alumínio e atuou como agente cimentante e coagulante das partículas tornando o solo mais poroso o que melhora a sua estrutura e favorece 0 desenvolvimento das plantas.
\end{abstract}

Palavras-chave: Resíduo de ETA, aproveitamento agrícola, disposição final.

\begin{abstract}
This paper aimed to evaluate the physical and chemical properties of an latosol after application of the sludge originated in the decanters of a water treatment plant. Five dosages of sludge were incorporated into the soil in four repetitions, one of which was without sludge, while the others were defined according to the concentration of nitrogen present in the sludge and in the soil, and according to plant demand (grass). The chemical and physical properties of the soil assessed before and after the sludge
\end{abstract}


application includes the concentration of $\mathrm{Al}^{+}{ }^{3}, \mathrm{pH}$, cation-exchange capacity, sum of the bases, aluminum and bases saturation, electrical conductivity, organic matter, nutrients $\mathrm{P}, \mathrm{K}, \mathrm{Ca}, \mathrm{Mg}, \mathrm{S}$, the metals $\mathrm{Cu}, \mathrm{Mn}, \mathrm{Fe}, \mathrm{Zn}, \mathrm{Cr}, \mathrm{Ni}, \mathrm{Cd}, \mathrm{Pb}$ and density. According to the findings, the sludge did not present characteristics indicating that this residue can be used as an agricultural corrective and fertilizer, and it was not possible to determine the best agronomic dose. The high concentration of $\mathrm{Mn}$ in the sludge limited the application of $2,5 \mathrm{mg} \mathrm{kg}^{-1}$. However, the sludge reduced by $20 \%$ the aluminum saturation index and acted as a luting agent and particle coagulant, making the soil more porous, which improves its structure, favoring the development of the plants.

Key-words: Water treatment residue, agricultural use, final disposal

\section{INTRODUÇÃO}

As Estações de Tratamento de Água (ETA) possuem funcionamento semelhante a uma indústria onde a matéria prima é transformada em produto final e durante esse processo são gerados resíduos chamados lodo de ETA. No Brasil, $62 \%$ do lodo de ETA são lançados em cursos de água sem qualquer tratamento, infringindo as leis de disposição de resíduos sólidos e do lançamento de efluentes em corpos d'água (IBGE, 2010).

De acordo com Tsutiya e Hidrata (2001), nos países do hemisfério norte, diferentes alternativas de uso benéfico do lodo têm sido adotadas transformando, em larga escala, resíduos em recursos. Na França 53\% do lodo gerado nas ETA são reaproveitados na construção civil e na compostagem (ADLER, 2002). Na Holanda, já em 1989, apenas $2 \%$ do lodo eram lançados em cursos d'água, $7 \%$ utilizados no controle de gás sulfídrico e $12 \%$ eram utilizados na agricultura (PEREIRA, 2011). Nos Estados Unidos, 25\% do lodo de ETA é utilizado como insumo agrícola e 11\% lançado no corpo hídrico (AWWARF, 1999).

O reaproveitamento agrícola do lodo de ETA torna-se interessante devido à presença de elementos importantes para as plantas como o cálcio e 0 magnésio (PADILHA, 2007). Teixeira et al. (2005) destacam também a utilização do lodo de ETA na recuperação de áreas degradadas em virtude do possível aumento de teores de macronutrientes e do $\mathrm{pH}$ do solo. Segundo Tsutiya e Hidrata (2001) às vantagens da aplicação do lodo de ETA inclui a melhoria estrutural do solo, ajuste do $\mathrm{pH}$, adição de traços minerais, aumento da capacidade de retenção de água e melhoria das condições de aeração, 
porém ressaltam que a disposição do resíduo requer monitoramento contínuo para controlar os nutrientes e metais pesados no solo.

Diante do exposto objetivou-se neste trabalho avaliar as propriedades de um latossolo após aplicação de lodo de ETA.

\section{MATERIAIS E MÉTODOS}

O experimento foi realizado no município de Juiz de Fora, estado de Minas Gerais, entre os agosto de 2014 e março de 2015. Uma descrição sucinta das etapas do experimento está demonstrada no Quadro 1.

Quadro 1: Etapas do experimento

\begin{tabular}{|c|c|c|c|c|}
\hline Etapa & Características & $\begin{array}{l}\mathrm{N}^{\circ} \text { de } \\
\text { amostras }\end{array}$ & $\begin{array}{l}N^{\circ} \text { de amostras } \\
\text { analisadas }\end{array}$ & Data \\
\hline Coleta do solo & Coleta das amostras de solo & 25 & 1 & $10 / 08 / 2014$ \\
\hline $\begin{array}{l}\text { Secagem do } \\
\text { solo }\end{array}$ & $\begin{array}{l}\text { Homogenização e secagem a } \\
\text { sombra das amostras de solo }\end{array}$ & - & - & $\begin{array}{c}11 / 08 \mathrm{a} \\
22 / 08 / 2014\end{array}$ \\
\hline $\begin{array}{l}\text { Caracterização } \\
\text { do solo }\end{array}$ & $\begin{array}{l}\text { Uma alíquota do solo foi enviada ao } \\
\text { Laboratório da UFV para } \\
\text { caracterização }\end{array}$ & - & 1 & $29 / 10 / 2014$ \\
\hline \multirow{2}{*}{ Coleta do lodo } & $\begin{array}{l}\begin{array}{l}\text { Coleta do lodo no } \\
\text { decantador }\end{array} \\
\end{array}$ & $1^{*}$ & \multirow{2}{*}{1} & $17 / 08 / 2014$ \\
\hline & $\begin{array}{l}\begin{array}{l}\text { Coleta do lodo no } \\
\text { decantador }\end{array} \\
\end{array}$ & $1^{*}$ & & $24 / 08 / 2014$ \\
\hline $\begin{array}{l}\text { Desidratação } \\
\text { do lodo }\end{array}$ & $\begin{array}{l}\text { Todas as amostras das duas coletas } \\
\text { do lodo foram homogeneizadas, } \\
\text { desidratadas e trituradas }\end{array}$ & - & - & $\begin{array}{c}18 / 08 \mathrm{a} \\
02 / 09 / 2014\end{array}$ \\
\hline $\begin{array}{l}\text { Caracterização } \\
\text { do lodo }\end{array}$ & $\begin{array}{l}\text { Após a desidratação } \\
\text { homogeneização das amostras do } \\
\text { lodo, uma alíquota foi enviada ao } \\
\text { Laboratório da UFV para } \\
\text { caracterização }\end{array}$ & - & 1 & $29 / 10 / 2014$ \\
\hline $\begin{array}{l}\text { Cálculo da } \\
\text { DRES }\end{array}$ & $\begin{array}{l}\text { Determinação dos tratamentos } \\
\text { (doses de lodo) a serem aplicadas } \\
\text { ao solo }\end{array}$ & - & - & $11 / 12 / 2014$ \\
\hline $\begin{array}{l}\text { Montagem do } \\
\text { experimento }\end{array}$ & $\begin{array}{l}\text { Montagem das unidades } \\
\text { experimentais utilizando vasos, brita } \\
\text { e a mistura de solo e lodo de acordo } \\
\text { com cada tratamento }\end{array}$ & 20 & - & $27 / 01 / 2015$ \\
\hline Irrigação & $\begin{array}{l}\text { Reposição da água perdida por } \\
\text { evaporação }\end{array}$ & 20 & - & $\begin{array}{c}28 / 01 \mathrm{a} \\
12 / 03 / 2015\end{array}$ \\
\hline Caracterização & Uma alíquota de cada unidade & 20 & 20 & $12 / 03 / 2015$ \\
\hline
\end{tabular}


do solo após experimental foi enviada ao

aplicação do Laboratório da UFV para

lodo

caracterização

Análise

estatística

Realizou-se Análise de Variança

dos resultados para um nível de

significância de $5 \%$

* Amostras integradas.

O solo foi coletado no município de Silveirânia, Minas Gerais em área onde há plantação de eucalipto. O solo da região é classificado como latossolo vermelho-amarelo (MAPA DE SOLOS DO ESTADO DE MINAS GERAIS, 2010).

A coleta foi realizada como sugere Santos et al. (2013). Todas as 25 amostras de solo foram misturadas e secadas a sombra e após homogeneização uma alíquota foi enviada ao Laboratório de Rotina e Física do Solo da Universidade Federal de Viçosa (UFV) para caracterização segundo EMBRAPA (2011) (Tabela 1).

Tabela 1: Caracterização química e física do solo

\begin{tabular}{cccc}
\hline Parâmetro & Valor & Parâmetro & Valor \\
\hline $\mathrm{pH}\left(\mathrm{em} \mathrm{H}_{2} \mathrm{O}\right)$ & 4,02 & $\mathrm{~S}\left(\mathrm{mg} \mathrm{dm}^{-3}\right)$ & 3,60 \\
$\mathrm{P}\left(\mathrm{mg} \mathrm{dm}^{-3}\right)$ & 0,70 & $\mathrm{~B}\left(\mathrm{mg} \mathrm{dm}^{-3}\right)$ & 0,25 \\
$\mathrm{~K}\left(\mathrm{mg} \mathrm{dm}^{-3}\right)$ & 13,0 & $\mathrm{Cu}\left(\mathrm{mg} \mathrm{dm}^{-3}\right)$ & 1,45 \\
$\mathrm{Ca}^{2+}\left(\mathrm{cmol} \mathrm{dm}^{-3}\right)$ & 0,07 & $\mathrm{Mn}\left(\mathrm{mg} \mathrm{dm}^{-3}\right)$ & 4,40 \\
$\mathrm{Mg}^{2+}\left(\mathrm{cmol} \mathrm{dm}^{-3}\right)$ & 0,04 & $\mathrm{Fe}\left(\mathrm{mg} \mathrm{dm}^{-3}\right)$ & 83,3 \\
$\mathrm{Al}^{3+}\left(\mathrm{cmol} \mathrm{dm}^{-3}\right)$ & 1,16 & $\mathrm{Zn}\left(\mathrm{mg} \mathrm{dm}^{-3}\right)$ & 0,45 \\
$\mathrm{H} \mathrm{Al}^{1}\left(\mathrm{cmol} \mathrm{dm}^{-3}\right)$ & 6,00 & $\mathrm{CE}^{7}\left(\mu \mathrm{s} \mathrm{cm}^{-1}\right)$ & 40,0 \\
$\mathrm{SB}\left(\mathrm{cmol} \mathrm{dm}^{-3}\right)$ & 0,14 & Areia grossa $(\%)$ & 26,0 \\
$\mathrm{t}^{2}\left(\mathrm{cmol} \mathrm{dm}^{-3}\right)$ & 1,30 & Areia fina $(\%)$ & 8,0 \\
$\mathrm{~T}^{3}\left(\mathrm{cmoldm}^{-3}\right)$ & 6,14 & Silte $(\%)$ & 9,0 \\
$\mathrm{~V}^{4}(\%)$ & 2,30 & Argila (\%) & 57,0 \\
$\mathrm{M}^{5}(\%)$ & 89,20 & $\rho^{8}\left(\mathrm{~g} \mathrm{~cm}^{-3}\right)$ & 1,05 \\
$\mathrm{MO}^{6}\left(\mathrm{dag} \mathrm{kg}^{-1}\right)$ & 2,69 & & \\
\hline
\end{tabular}

1 Acidez potencial; 2 Capacidade de troca de cátions (CTC) efetiva $=\mathrm{SB}+\mathrm{Al}^{3^{+}} ; 3 \mathrm{CTC}$ pH 7 = SB+ (H+Al); 4 Índice de saturação de bases; 5 Índice de saturação de alumínio; 6 Matéria orgânica; 7 Condutividade elétrica; 8 Densidade.

O lodo foi obtido de uma estação de tratamento de água, com tecnologia de tratamento de ciclo completo que trata $620 \mathrm{~L} \mathrm{~s}^{-1}$ utilizando cal como corretor de $\mathrm{pH}$ e coagulante Floculan. Coletou-se o lodo do decantador devido a sua maior concentração de sólidos. A ETA opera com dois decantadores convencionais de $1.609 \mathrm{~m}^{3}$, que são lavados manualmente. Para a 
caracterização do lodo ocorreram duas coletas de amostras nos dias 17 e 24 de agosto de 2014. Em cada coleta, a amostragem foi do tipo integrada, sendo representativa de todo o decantador (os dois decantadores foram amostrados, sendo um em cada coleta).

As amostras de lodo foram desidratadas em estufa até um teor de umidade constante e posteriormente foi triturado. O lodo obtido nas duas coletas, após a desidratação, foi homogeneizado e enviado ao Laboratório da UFV para caracterização segundo EMBRAPA (2011) (Tabela 2).

Tabela 2: Caracterização do lodo da ETA

\begin{tabular}{cccccc}
\hline Parâmetro & Valor & Parâmetro & Valor & Parâmetro & Valor \\
\hline $\mathrm{Ca}\left(\mathrm{g} \mathrm{kg}^{-1}\right)$ & 51,9 & $\mathrm{Fe}\left(\mathrm{mg} \mathrm{kg}^{-1}\right)$ & 321,1 & $\mathrm{~Pb}\left(\mathrm{mg} \mathrm{kg}^{-1}\right)$ & 6,4 \\
$\mathrm{Mg}\left(\mathrm{g} \mathrm{kg}^{-1}\right)$ & 32,1 & $\mathrm{Zn}\left(\mathrm{mg} \mathrm{kg}^{-1}\right)$ & 155,3 & $\mathrm{Ni}\left(\mathrm{mg} \mathrm{kg}^{-1}\right)$ & 71,5 \\
$\mathrm{~N}\left(\mathrm{~g} \mathrm{~kg}^{-1}\right)$ & 21,0 & $\mathrm{Mn}\left(\mathrm{mg} \mathrm{kg}^{-1}\right)$ & 525,9 & $\mathrm{~B}\left(\mathrm{mg} \mathrm{kg}^{-1}\right)$ & 3,1 \\
$\mathrm{P}\left(\mathrm{g} \mathrm{kg}^{-1}\right)$ & 22,9 & $\mathrm{Cu}\left(\mathrm{mg} \mathrm{kg}^{-1}\right)$ & 37,5 & $\mathrm{~S}\left(\mathrm{mg} \mathrm{kg}^{-1}\right)$ & 2,45 \\
$\mathrm{~K}\left(\mathrm{~g} \mathrm{~kg}^{-1}\right)$ & 14,6 & $\mathrm{Cd}\left(\mathrm{mg} \mathrm{kg}^{-1}\right)$ & 11,2 & $\mathrm{PN}^{1}(\%)$ & 1,2 \\
$\mathrm{MO}\left(\mathrm{g} \mathrm{kg}^{-1}\right)$ & 401,0 & $\mathrm{Cr}\left(\mathrm{mg} \mathrm{kg}^{-1}\right)$ & 105,5 & $\mathrm{ST}^{\star}\left(\mathrm{mg} \mathrm{L}^{-1}\right)$ & $101.286,3$ \\
\hline \multicolumn{5}{c}{ 1 Poder neutralizante. } \\
* Teor de sólidos totais foi determinado segundo APHA (2005) à temperatura de 105 ${ }^{\circ} \mathrm{C}$ \\
por uma hora.
\end{tabular}

De acordo com a caracterização do solo e do lodo foi possível estabelecer a dose de aplicação do resíduo, calculada conforme a Equação 1 (MATOS, 2007).

Equação 1:

$$
\mathrm{D}_{\text {res }}=\frac{\mathrm{N}_{\mathrm{abs}}-\mathrm{T}_{\mathrm{m} 1} \cdot \mathrm{MO} \cdot \mathrm{\rho}_{\mathrm{s}} \cdot \mathrm{p} \cdot 10^{7} \cdot 0,05 \cdot \frac{\mathrm{n}}{12}}{\mathrm{~T}_{\mathrm{m} 2} \cdot \frac{\mathrm{n}}{12} \cdot \mathrm{N}_{\text {org }}+\left(\mathrm{N}_{\text {amon }}+\mathrm{N}_{\text {nitrato }}\right) \cdot \mathrm{TR}}
$$

Onde:Dres - dose de resíduo (t ha-1); $\mathrm{N}_{\text {abs }}$ - nitrogênio absorvido pela cultura ( $\left.\mathrm{kg} \mathrm{ha}^{-1}\right) ; \mathrm{T}_{\mathrm{m} 1}$ - taxa anual de mineralização da matéria orgânica do solo $\left(\mathrm{kg} \mathrm{kg}^{-1}\right.$ ano-1); MO - matéria orgânica do solo $\left(\mathrm{kg} \mathrm{kg}^{-1}\right)$; $\rho_{\mathrm{s}}$ - densidade do solo $\left(\mathrm{t} \mathrm{m}^{-3}\right) ; \mathrm{p}$ - profundidade do solo $(\mathrm{m}) ; \mathrm{n}$ - número de meses do ano; Tm2 - taxa de mineralização do $\mathrm{N}_{\mathrm{org}}\left(\mathrm{kg} \mathrm{kg}^{-1}\right.$ ano-1); TR - proporção na recuperação do $\mathrm{N}$ mineral pela cultura $\left(\mathrm{kg} \mathrm{kg}^{-1}\right)$; Norg - nitrogênio orgânico $\left(\mathrm{g} \mathrm{kg}^{-1}\right)$; Nanom nitrogênio amoniacal $\left(\mathrm{g} \mathrm{kg}^{-1}\right)$; Nnitrato - nitrato $\left(\mathrm{g} \mathrm{kg}^{-1}\right)$.

$\mathrm{T}_{\mathrm{m} 1}$ varia, geralmente, entre 0,01 e 0,02 . $\mathrm{O}$ valor utilizado foi de 0,01 . A profundidade adotada foi de $0,20 \mathrm{~m}$ e o o número de meses considerados foram 12. Não foi encontrado na literaruta valores referentes a taxa de 
mineralização do $N_{\text {org }}\left(T_{m}\right)$ para o lodo de ETA sendo utilizado o valor de 0,3 $\mathrm{kg} \mathrm{kg}^{-1}$ ano-1 $^{-1}$ que corresponde a taxa anual de mineralização do material orgânico do lodo de esgoto digerido aerobicamente (MATOS, 2007).

Considerou-se a adubação de gramínea para determinação do $\mathrm{Nabs}_{\text {, }}$ sendo este igual a $200 \mathrm{~kg} \mathrm{ha}^{-1}$ (RIBEIRO et al., 1999a). Segundo Megda et al. (2005), os lodos de ETA podem ser aplicados no cultivo de grama durante a preparação do solo e na fase de crescimento da planta. Para gramíneas o TR é de $0,5 \mathrm{~kg} \mathrm{~kg}^{-1}$ (MATOS, 2007).

A concentração média de nitrato em lodo de ETA obtido por Andreoli et al. (2006), determinada através da análise de 71 trabalhos, foi de $0,2 \mathrm{~g} \mathrm{~kg}^{-1}$. Ippolito et al. (2010), por meio da avaliação de 21 estudos, aferiram o valor de $0,02 \mathrm{~g} \mathrm{~kg}^{-1}$ de nitrogênio amoniacal.

Após calcular a Dresdo lodo a ser aplicado, definiu-se variar as propoções de $0 \%, 50 \%, 100 \%, 150 \%$ e $200 \%$ desta dose (Tabela 3 ).

Tabela 3: Tratamentos em função da dose de aplicação do lodo

\begin{tabular}{cccc}
\hline & Proporção da Dres & Proporção da Dres & Taxa de aplicação \\
\cline { 2 - 4 } & $\%$ & $\mathrm{~g} \mathrm{vaso}^{-1}$ & $\mathrm{~kg} \mathrm{~m}^{-2}$ \\
\hline Tratamento 0 & 0 & 0,00 & 0,00 \\
Tratamento 1 & 50 & 29,14 & 1,36 \\
Tratamento 2 & 100 & 58,27 & 2,73 \\
Tratamento 3 & 150 & 87,41 & 4,09 \\
Tratamento 4 & 200 & 166,55 & 5,45 \\
\hline
\end{tabular}

O experimento seguiu um delineamento inteiramente casualizado (DIC) com cinco tratamentos (doses de lodo) e quatro repetições, totalizando 20 unidades experimentais (Figura 1).

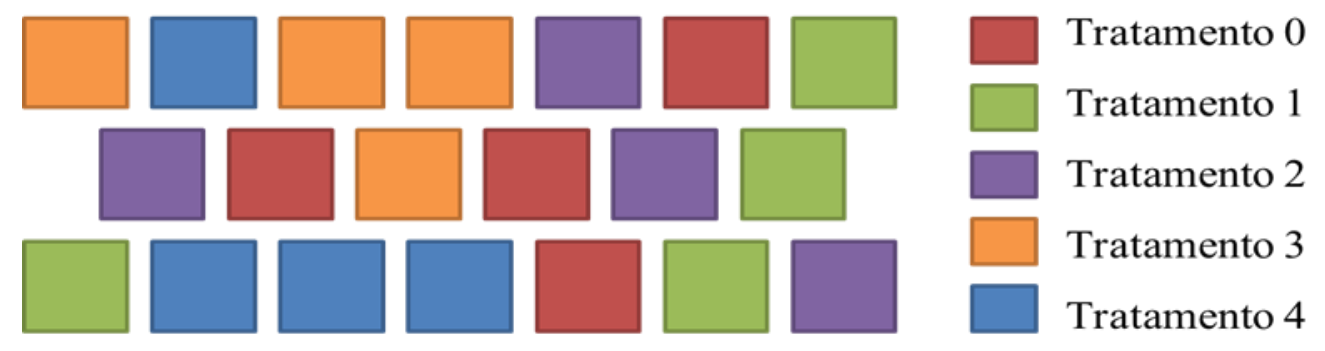


Figura 1. Disposição dos vasos conforme o DIC.

Para montagem das unidades experimentais utilizou-se vasos de polietileno preto com capacidade de $3 \mathrm{~L}$. Cada vaso recebeu uma camada de brita no fundo para favorecer a drenagem e aeração do solo. Em seguida recebeu $2 \mathrm{~kg}$ da mistura de solo mais lodo nas proporções determinadas para cada tratamento.

Cada vaso foi umedecido diariamente com água destilada a fim de manter a umidade do solo próxima à sua capacidade de campo. A reposição de água foi calculada segundo a Equação 2 (TUCCl et al., 1993).

Equação 2:

$$
E V=0,35 \cdot\left(1+0,24 \cdot u_{2}\right) \cdot\left(e_{s}-e_{a}\right)
$$

Sendo: EV - evaporação da superficie $\left(\mathrm{mm} \mathrm{dia}^{-1}\right)$; es - umidade de saturação do ar (mb) (Equação 3); $e_{a}$ - umidade de vapor de água a temperatura ambiente $(\mathrm{mb})$ (Equação 4 ); $\mathrm{u}_{2}$ - velocidade do vento a $2 \mathrm{~m}$ de altura $\left(\mathrm{m} \mathrm{s}^{-1}\right)$ e $\mathrm{T}$ - temperatura média do dia em ${ }^{\circ} \mathrm{C}$.

Equação 3:

$$
\mathrm{e}_{\mathrm{s}}=0,61 \cdot \exp \left(\frac{17,27 . \mathrm{T}}{237,3+\mathrm{T}}\right)
$$

Equação 4:

$$
\mathrm{e}_{\mathrm{a}}=\mathrm{e}_{\mathrm{s}} \frac{\mathrm{UR}}{100}
$$

Em que: UR - umidade relativa do ar média mensal (\%) e es em $\mathrm{kPa}$.

Para a determinação da evaporação utilizou-se os dados da estação meteorológica Juiz de Fora obtidos no portal do Instituto Nacional de Meteorologia (INMET, 2015).

Para avaliar o efeito da aplicação do lodo de ETA, amostras foram coletadas após 44 dias do início do experimento e caracterizadas segundo EMBRAPA (2011). Dos atributos químicos e físicos do solo foram avaliados a concentração de $\mathrm{Al}^{+3}, \mathrm{pH}, \mathrm{CTC}, \mathrm{SB}, \mathrm{V}, \mathrm{m}, \mathrm{CE}, \mathrm{MO}, \rho, \mathrm{P}, \mathrm{K}, \mathrm{Ca}, \mathrm{Mg}, \mathrm{S}, \mathrm{Cu}, \mathrm{Mn}$, $\mathrm{Fe}, \mathrm{Zn}, \mathrm{Cr}, \mathrm{Ni}, \mathrm{Cd}$ e $\mathrm{Pb}$. 
Realizou-se Análise de Variança (ANOVA), considerando duas hipóteses: $\mathrm{H}_{0}$ - as características do solo não são alteradas em função da dosagem do lodo e $\mathrm{H}_{1}$ - as características do solo são alteradas em função da dosagem do lodo, para um nível de significancia de 5\%. A ANOVA foi realizada com o auxílio do softwear SISVAR, desenvolvido por Ferreira (2003). Utilizouse o softwear SigmaPlot para o ajuste dos modelos de regressão das características do solo avaliadas em função da dose, quando aceitou-se $\mathrm{H}_{1}$.

\section{RESULTADOS E DISCUSÕES}

Os parâmetros que variaram, considerando um nível de probabilidade de $5 \%$ para aceitar $\mathrm{H}_{1}$, foram: $\mathrm{Ca}^{2+}, \mathrm{Mg}^{2+}, \mathrm{SB}, \mathrm{V}, \mathrm{t}, \mathrm{m}, \mathrm{Fe}, \mathrm{Mn}, \mathrm{Zn}, \mathrm{Cr}, \mathrm{CE}$ e densidade. Os outros parâmetros: $\mathrm{pH}, \mathrm{P}, \mathrm{K}, \mathrm{Al}, \mathrm{MO}, \mathrm{Cu}, \mathrm{Ni}, \mathrm{Cd}$ e $\mathrm{Pb}$ não variaram estatisticamente de acordo com os resultados encontrados (Tabela 4).

Tabela 4: Concentrações médias dos parametros que não variaram em função dos tratamentos

\begin{tabular}{cccccc}
\hline Parâmetro & $\mathrm{T} 0$ & $\mathrm{~T} 1$ & $\mathrm{~T} 2$ & $\mathrm{~T} 3$ & $\mathrm{~T} 4$ \\
\hline $\mathrm{pH}\left(\mathrm{em} \mathrm{H}_{2} \mathrm{O}\right)$ & 5,16 & 5,00 & 4,79 & 4,81 & 4,67 \\
$\mathrm{P}\left(\mathrm{mg} \mathrm{dm}^{-3}\right)$ & 1,13 & 0,98 & 1,13 & 1,03 & 1,03 \\
$\mathrm{~K}\left(\mathrm{mg} \mathrm{dm}^{-3}\right)$ & 19,75 & 19,25 & 19,00 & 20,00 & 19,50 \\
$\mathrm{Al}{ }^{3+}\left(\mathrm{cmol} \mathrm{dm}^{-3}\right)$ & 0,88 & 1,00 & 1,05 & 0,90 & 0,86 \\
$\mathrm{H}+\mathrm{AL}\left(\mathrm{cmol} \mathrm{dm}^{-3}\right)$ & 7,93 & 7,08 & 6,60 & 6,28 & 6,95 \\
$\mathrm{~T}\left(\mathrm{cmol} \mathrm{dm}^{-3}\right)$ & 8,26 & 7,54 & 7,14 & 6,93 & 7,71 \\
$\mathrm{MO}\left(\mathrm{dag} \mathrm{kg}^{-1}\right)$ & 3,36 & 3,40 & 3,26 & 3,43 & 3,49 \\
$\mathrm{Cu}\left(\mathrm{mg} \mathrm{dm}^{-3}\right)$ & 1,73 & 1,69 & 1,78 & 1,99 & 1,98 \\
$\mathrm{Ni}\left(\mathrm{mg} \mathrm{dm}^{-3}\right)$ & 0,03 & 0,31 & 0,01 & 0,39 & 0,25 \\
$\mathrm{Cd}\left(\mathrm{mg} \mathrm{dm}^{-3}\right)$ & 0,03 & 0,03 & 0,04 & 0,01 & 0,06 \\
$\mathrm{~Pb}\left(\mathrm{mg} \mathrm{dm}^{-3}\right)$ & 0,68 & 0,56 & 0,69 & 0,56 & 0,58 \\
\hline
\end{tabular}

No que diz respeito a classificaçao agronômica o teor de matéria orgânica (MO) é médio (de 2 a $4 \mathrm{cmol} \mathrm{dm}^{-3}$ ) em todos os tratamentos, sendo essa a mesma classificação para a CTC a pH 7 (T) (de 4,3 a 8,6 $\mathrm{cmol} \mathrm{dm}^{-3}$ ) (RIBEIRO et al., 1999a) uma vez que a MO pode representar mais de $80 \%$ do valor da CTC (RONQUIM, 2010).

A acidez ativa $(\mathrm{pH})$ e potencial $(\mathrm{H}+\mathrm{AL})$ manteve-se alta (menor que 5; entre 5 e $9 \mathrm{cmol} \mathrm{dm}^{-3}$ respectivamente) (RIBEIRO et al., 1999a). Apesar da ETA onde o lodo foi coletado utilizar cal como corretor de $\mathrm{pH}$ da água, não houve variação na acidez do solo após a adubação com o resíduo para as 
taxas de aplicação adotadas. Acredita-se que a quatidade de cal utilizada na ETA é reduzida e portanto não foi capaz de proporcionar ao lodo um poder de neutralização elevado, como pode ser observado na Tabela 2 este valor é de $1,2 \%$. Padilha (2007) também observou que o lodo de ETA não modificou o pH do latossolo vermelho-amarelo, permanecendo em 4,8.

A acidez trocável $\left(\left.A\right|^{3+}\right)$ oscilou entre a classificação média (entre 0,5 e $0,9 \mathrm{cmol} \mathrm{dm}^{-3}$ ) e alta (entre 1 e $2 \mathrm{cmol} \mathrm{dm}^{-3}$ ). A ETA não utiliza coagulate a base de alumínio e portanto a aplicação do lodo não alterou estatisticamente as concentrações desse elemento no solo. Os teores elevados de Al podem estar relacionados a composição natural do solo, em latossolo vermelho-amarelo os óxidos de alumínio são os responsáveis pela coloração amarela (KER, 1991), o Al pode também ter origem na decomposição de argilas caoliníticas que liberam Al ${ }^{3+}$ (RONQUIN, 2010). Agyin-Birikorang, et al. (2009) também verificaram não haver alterações nas concentraçõesde Al no solo após aplicação de lodo de ETA.

Considerando a densidade média do solo de $1,05 \mathrm{~g} \mathrm{~cm}^{-3}$, os teores de $\mathrm{Cu}, \mathrm{Ni}, \mathrm{Cd}$ e $\mathrm{Pb}$ atendem a CONAMA 420/2009 que dispõe sobre critérios e valores orientadores de qualidade do solo quanto à presença de substâncias químicas (BRASIL, 2009), a legislação norte americana 40 CFR Part 503 que estabelece padrões para a utilização de lodo de estação de tratamento de esgoto (ETE) e a Directive Europeia 86/278/EEC que trata da proteção do solo quando utiliza-se lodo de ETE na agricultura (USEPA, 1996).

Os resultados determinados nesse estudo são semelhantes aos obtidos por Bittencourt et al. (2012) que também concluiram que o lodo aplicado ao solo, em diferentes doses, não variou os teores de Cu (manteve-se próximo de $23 \mathrm{mg} \mathrm{kg}^{-1}$ ) e $\mathrm{Pb}$ (igual $20 \mathrm{mg} \mathrm{kg}^{-1}$ ).

$\mathrm{Na}$ Figura 2A e 2B apresentam-se, respectivamente, as variação das concentrações de $\mathrm{Ca}^{2+} \mathrm{e} \mathrm{Mg}^{2+}$ em função da taxa de aplicação de lodo da ETA. 

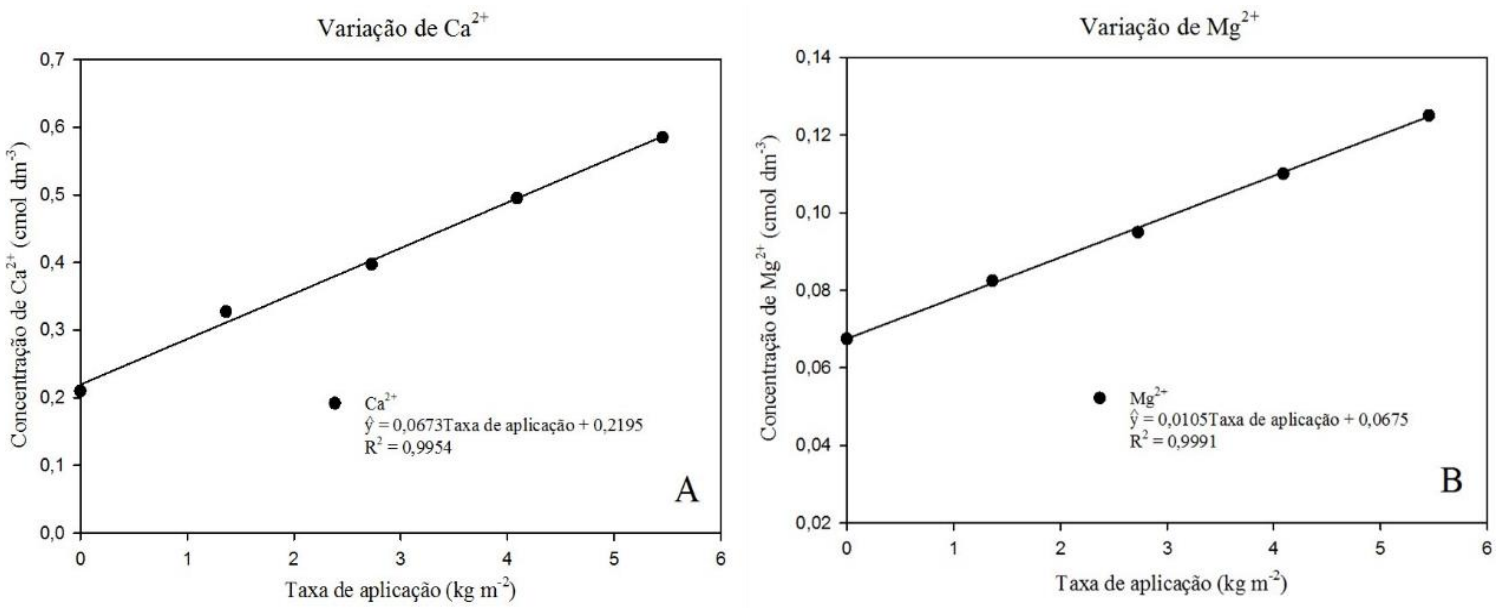

Figura 2. Curva de variação das concentrações de $\mathrm{Ca}^{2+}$ e $\mathrm{Mg}^{2+}$ em função da taxa de aplicação de lodo da ETA

As concentrações de $\mathrm{Ca}^{2+}$ e $\mathrm{Mg}^{2+}$ aumentaram proporcionalmente à quantidade de lodo de ETA (), porém mesmo após o tratamento T4 não foi possível suprir a deficiência desses nutrientes atingindo ao menos os valores recomendados de $2,0 \mathrm{cmol} \mathrm{dm}^{-3}$ de $\mathrm{Ca}^{2+}$ e $0,5 \mathrm{cmol} \mathrm{dm}^{-3}$ de $\mathrm{Mg}^{2+}$ (SBCS, 2004).

Teixeira et al. (2005) observaram um aumento de 0,34 para $12,10 \mathrm{~g} \mathrm{~kg}^{-1}$ de cálcio total e 0,10 para $0,46 \mathrm{~g} \mathrm{~kg}^{-1} \mathrm{de}$ magnésio total quando compararam o solo sem aplicação e com aplicação de $200 \mathrm{mg} \mathrm{kg}^{-1}$ de nitrogênio na forma de lodo da ETA Araraquara. Bittencourt et al. (2012), no entanto, concluiram que as diferentes doses de lodo da ETA Passaúna não alteraram as concentrações de $\mathrm{Ca}$ e $\mathrm{Mg}$ no solo para um nível de $5 \%$ de probabilidade.

Na Figura 3A e 3B apresentam-se os valores médios para os parâmetros soma de bases (SB) e capacidade de troca de cátions (CTC) efetiva.

$O$ aumento da SB reflete o aumento nas concentrações de $\mathrm{Ca}^{2+} \mathrm{e} \mathrm{Mg}^{2+}$ assim como na CTC efetiva. A matéria orgânica presente no lodo também pode ter contribuído no aumento da CTC. Moreira (2013) concluiu que o maior incremento na CTC foi obtido com a maior dose de lodo de ETE aplicado no solo devido a elevação do teor de MO. 

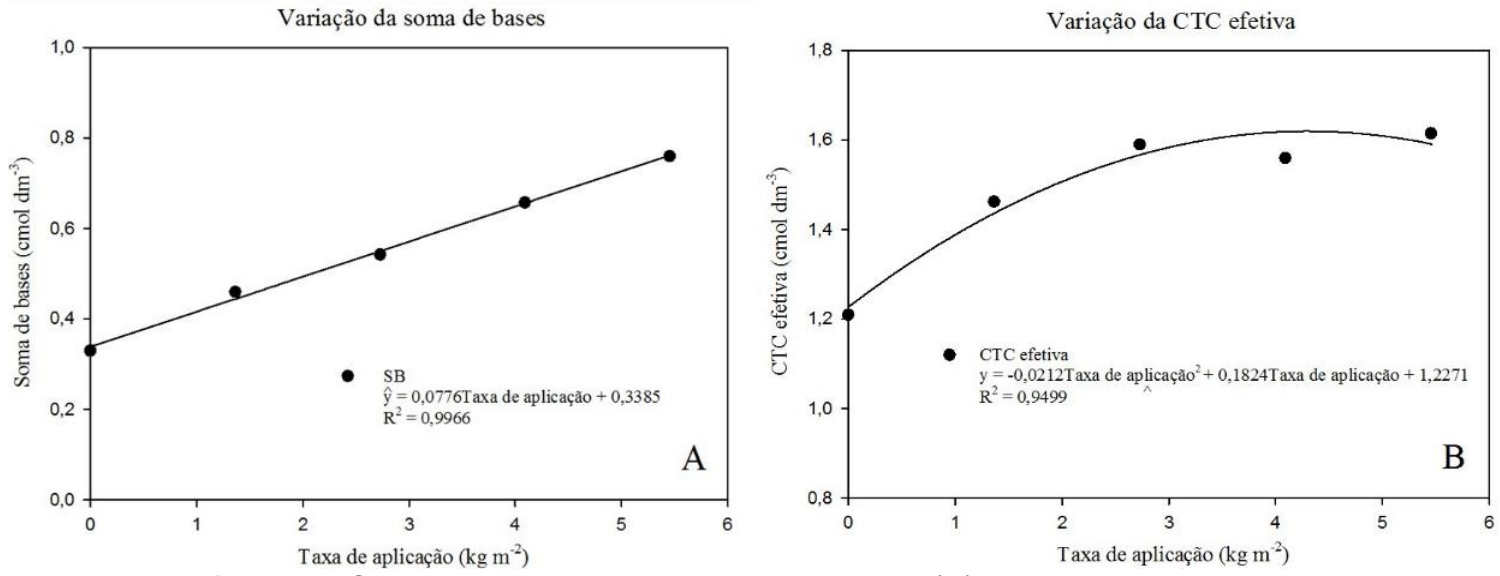

Figura 3. Curva de variação da soma de bases $(A)$ e da capacidade de troca de cátions(CTC) efetiva (B) em função da taxa de aplicação de lodo da ETA

Segundo Ribeiro et al. (1999a) a classificação para a SB é muito baixa nos tratamentos T0, T1 e T2 e baixa no T3 e T4, de acordo com o mesmos autores a soma de bases deve ser maior que $3,6 \mathrm{cmol} \mathrm{dm}^{-3}$, este valor não foi atingido com as taxas de aplicação adotadas neste estudo, o valor máximo foi de $0,76 \mathrm{cmol} \mathrm{dm}^{-3}$.

A CTC efetiva pernameceu em todos os tratamentos na classe baixa, sendo que valores superiores a $4,6 \mathrm{cmol} \mathrm{dm}^{-3}$ indicam boa fertilidade do solo (RIBEIRO et al., 1999a). Assim como a SB, a CTC efetiva não alcançou esta concentração nos tratamentos adotados e limitou-se a $1,62 \mathrm{cmol} \mathrm{dm}^{-3}$.

$\mathrm{Na}$ Figura 4 encontram-se os resultados para o índice de saturação por bases (V) e o índice de saturação por alumínio (m).

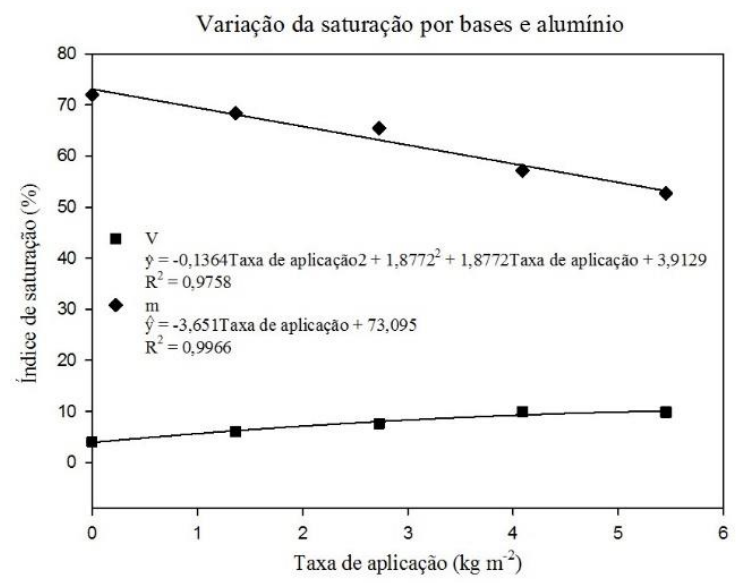

Figura 4. Variação do índice de saturação por bases (V) e por alumínio (m) em função da taxa de aplicação de lodo da ETA 
Nota-se que houve um aumento do índice de saturação por bases proporcional a dose de lodo aplicado evidênciando que o lodo foi capaz de fornecer cátions bases, no entanto os altos índices de saturação por alumínio (maior que 50\%) indicam que a CTC efetiva está ocupada, em maior parte, pelo alumínio trocável. Krob et al. (2011) também observaram uma redução de alumínio devido a adição dos cátions trocáveis $\mathrm{Ca}$ e $\mathrm{Mg}$ e aumento da SB com a aplicação de lixo urbano compostado.

Apesar do aumento da saturação por bases, esta não atingiu os valores recomendados para algumas gramíneas que se desenvolvem em um mínimo de $40 \%$ de $V$ (RIBEIRO et al.1999b). Segundo Ribeiro et al. (1999a) o solo possui $\mathrm{V}$ muito baixo e mesmo após o tratamento T4 manteve-se classificado como solo distrófico.

Para a saturação por alumínio houve uma redução de $20 \%$ se comparados os tratamentos T0 e T4, mas o m ainda é alto para todos os tratamentos. O índice deve ser inferior a 15\% para indicar nível não tóxico de $\mathrm{Al}$, no entanto a tolerância varia para cada cultura, o eucalipto suporta até $45 \%$, o capim-limão tolera $25 \%$ e os gramandos até $5 \%$ (RIBEIRO et al. 1999a).

$\mathrm{Na}$ Figura 5 apresentam-se os valores médios para o Zn (Figura 5A), o $\mathrm{Cr}$ (Figura 5B), o Mn (Figura 5C) e o Fe (Figura 5D) nos diferentes tratamentos.

Figura 5. Variação da concentração de zinco (A), cromo (B), manganês (C) e ferro (D) em função da taxa de aplicação de lodo da ETA

As concentrações desses metais variaram proporcionalmente a aplicação do lodo. Essa elevação está relacionada com o aumento da CTC, pois mais sítios de troca do solo estarão disponíveis para a retenção de metais (MOREIRA, 2013).

Para Matos (2014) o lodo de ETA possui cerca de $76,0 \mathrm{mg} \mathrm{kg}^{-1}$ de $\mathrm{Zn}$, Teixeira et al. (2005) obteve $66,0 \mathrm{mg} \mathrm{kg}^{-1}$ de $\mathrm{Zn}$ para o lodo de ETA cujo tratamento é do tipo convencional e utiliza cloreto férrico como coagulante, Padilha (2007) determinou o valor de $100,0 \mathrm{mg} \mathrm{kg}^{-1}$ de $\mathrm{Zn}$ no lodo de uma estação que utiliza sulfato de alumínio como coagulante. Quando comparado esses resultados com o zinco presente no lodo em estudo nota-se que este 
elemento está em concentração elevada $\left(155,3 \mathrm{mg} \mathrm{kg}^{-1}\right)$ justificando assim o aumento dos teores de zinco no solo.

Segundo Ribeiro et al.(1999a) o Zn passou de baixo (entre 0,5 e 0,9 mg $\mathrm{dm}^{-3}$ ) para médio (entre 1,0 e 1,5 $\mathrm{mg} \mathrm{dm}^{-3}$ ) nos tratamentos T2, T3 e T4. Os mesmos autores afirmam que solos com boa fertilidade apresentam concentrações de zinco menores que $2,2 \mathrm{mg} \mathrm{dm}^{-3}$. De acordo com a Figura $5 \mathrm{~A}$ essa concentração não foi alcançada para uma taxa de aplicação de lodo de até $5,45 \mathrm{~kg} \mathrm{~m}^{-2}$ (T4).

Bertoncini (2002) aplicou lodo de ETE em duas doses, 0 e $388 \mathrm{t} \mathrm{ha}^{-1}$, e observou que o lodo adicionou ao solo $947 \mathrm{~kg}$ de $\mathrm{Zn}$. Basso et al (2012) determinaram os teores de metais pesados no solo após aplicação de dejeto líquido de suínos e concluiram que o $\mathrm{Zn}$ e $\mathrm{Cu}$ são os elementos com maior tendência de acúmulo em áreas que recebem sucessivas aplicações do dejeto.

O cromo também encontra-se em concentração elevada $\left(105,5 \mathrm{mg} \mathrm{kg}^{-1}\right)$ se comparado à outros resultados como os de Bittencourt et al. (2012) que obtiveram valores menores que $2,0 \mathrm{mg} \mathrm{kg}^{-1}$ para o lodo da ETA que utiliza sulfato de alumínio como coagulante ou policloreto de alumínio e Teixeira et al. (2005) que determinou 86,0 $\mathrm{mg} \mathrm{kg}^{-1}$ de $\mathrm{Cr}$.

Merlino et al. (2010) avaliou a presença de bário, cádmio, cromo e chumbo em latossolo após onze aplicações anuais de lodo de esgoto e observou que o $\mathrm{Cr}$ foi o metal que apresentou maior concentração no solo com média de $88,3 \mathrm{mg} \mathrm{kg}^{-1}$.

Para Malavolta (1980) o efeito tóxico do Cr é observado para valores acima de $5 \mathrm{mg} \mathrm{dm}^{-3}$, como está apresentadona Figura 5B o cromo não atingiu esse valor no T4, exibindo comportamento semelhante ao zinco. Ambos os compostos, $\mathrm{Zn}$ e $\mathrm{Cr}$, apresentaram as maiores concentrações dentre os trabalhos pesquizados. No entanto, estes elementos atendem a CONAMA 420/2009 após aplicação no solo (BRASIL, 2009).

Em relação ao managês Bittencourt et al. (2012) obteve $649,0 \mathrm{mg} \mathrm{kg}^{-1}$; Padilha (2007) obteve $770,0 \mathrm{mg} \mathrm{kg}^{-1}$ e Teixeira et al. (2005) obteve $1.683,0 \mathrm{mg}$ $\mathrm{kg}^{-1}$ de $\mathrm{Mn}$ no lodo de ETA. Esses valores são menores que os $526,0 \mathrm{mg} \mathrm{kg}^{-1}$ de $\mathrm{Mn}$ no resíduo da ETA, no entanto este é o metal em maior concentração no lodo analisado, o que favoreceu o acréscimo de managês no solo. 
Após aplicação do lodo no solo, Ribeiro et al. (1999a) defini o teor de manganês como médio (entre 6 e $8 \mathrm{mg} \mathrm{dm}^{-3}$ ) no tratamento T0, bom (entre $9 \mathrm{e}$ $12 \mathrm{mg} \mathrm{dm}^{-3}$ ) no T1 e T2 e alto (maior que $12 \mathrm{mg} \mathrm{dm}^{-3}$ ) nos tratamentos T3 e T4. Em solos férteis o Mn encontra-se em valores menores que $12 \mathrm{mg} \mathrm{dm}^{-3}$, assim a taxa de aplicação de lodo da ETA não deve superar $2,5 \mathrm{~kg} \mathrm{~m}^{-2}$ conforme equação apresentada na Figura 5C.

Assim como o managês, o ferro presente no lodo da ETA está em menor concentração se comparado com os resultados de Bittencourt et al. (2012), Padilha (2007) e Teixeira et al. (2005), porém o solo analisado possui naturalmente um teor elevado de $\mathrm{Fe}$ com $83,3 \mathrm{mg} \mathrm{dm}^{-3}$ e a aplicação de lodo contribuiu no aumento. A textura argilosa do solo favorece ainda a retenção desse metal, permanecendo na classe alta (maior que $45 \mathrm{mg} \mathrm{dm}^{-3}$ ) em todos os tratamentos, indicando um nível crítico (RIBEIRO et al., 1999a).

Diferente dos resultados encontrados nesse trabalho para o $\mathrm{Zn}, \mathrm{Cr}, \mathrm{Mn}$ e $\mathrm{Fe}$, Bittencourt et al. (2012) concluiram que as diferentes doses de lodo de ETA não alteraram, estatisticamente, esses metais no solo.

Na Figura 6 apresenta-se os valores médios para a CE.

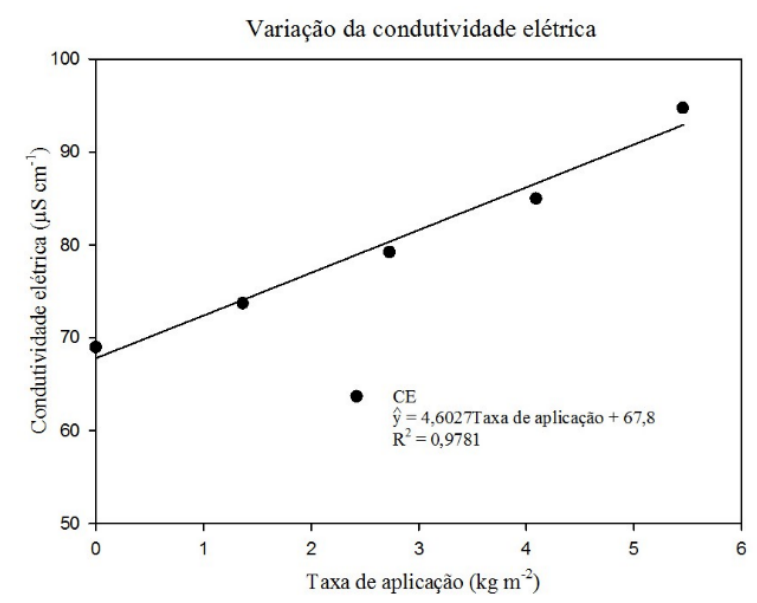

Figura 6. Curva de variação da condutividade elétrica (CE) em função da taxa de aplicação de lodo da ETA

Observa-se que a CE aumentou com a aplicação do lodo, este comportamento pode ser explicado devido à elevação dos cátions $\mathrm{Ca}^{2+}, \mathrm{Mg}^{2+} \mathrm{e}$ $\mathrm{Fe}^{3+}$.

O aumento da condutividade elétrica após tratamento com lodo de ETA também foi observado por Mahdy et al. (2013) quando aplicaram 0, 20, 40 e 80 
$\mathrm{g} \mathrm{kg}^{-1}$ de lodo e após 20 dias de incubação a CE foi de 846,5; 950,9; 1.245,0 e $1.347,0 \mu \mathrm{S} \mathrm{cm}^{-1}$ respectivamente.

Teixeira et al. (2005) alertam para o risco de salinização devido a aplicação do lodo de ETA e observaram que a CE passou de 370 para até $2.960 \mu \mathrm{S} \mathrm{cm}^{-1}$.

A condutividade elétrica acima de $4.000 \mu \mathrm{S} \mathrm{m}^{-1}$ é dita alta (BRADY e WEIL, 2013), porém algumas culturas são sensíveis a CE próximas de 1.000 $\mu S \mathrm{~cm}^{-1}$ (RIBEIRO et al., 1999a). De acordo com a Figura 7 o valor máximo alcançado foi de $95 \mu \mathrm{S} \mathrm{cm}^{-1}$, portanto bem abaixo do considerado alto.

$\mathrm{Na}$ Figura 7 apresenta-se os valores médios para a densidade nos diferentes tratamentos.

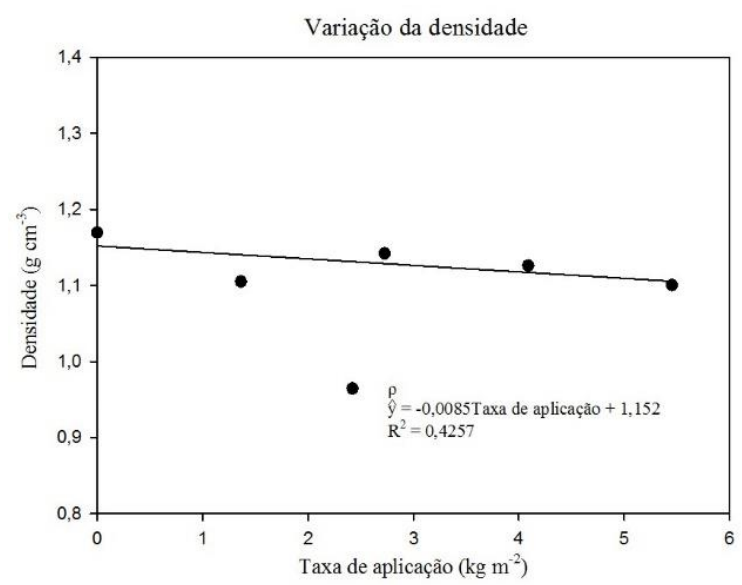

Figura 71. Variação da densidade em função da taxa de aplicação de lodo da ETA

De acordo com as equações de regressão a aplicação de lodo da ETA diminuiu a densidade do solo que passou de $1,17 \mathrm{~g} \mathrm{~cm}^{-3}$ no T0 para $1,10 \mathrm{~g} \mathrm{~cm}^{-3}$ no T4. Acredita-se que o ferro presente no lodo agiu como coagulante das partículas formando agregados e tornando o solo mais poroso. Este aumento da porosidade favorece a penetração das raízes, a aeração, a taxa de infiltração e a difusão do oxigênio, melhorando assim o desenvolvimento das plantas e a estrutura do solo.

A MO também pode atuar como agente cimentante das partículas e reduzir a densidade, como observado por Moreira (2013) que avaliou a aplicação de lodo de ETE no solo e concluiu que com a aplicação de $180 \mathrm{t} \mathrm{ha}^{-1}$ houve melhora da estrutura do solo, diminuindo a densidade de 1,38 para 1,06 $\mathrm{g} \mathrm{cm}^{-3}$. 


\section{CONCLUSÃO}

Para as condições nas quais o estudo foi desenvolvido, conclui-se que do ponto de vista agronômico o lodo da ETA foi incapaz de elevar os teores das bases aos níveis recomendados para solos férteis e não permitiu determinar a melhor dose agronômica; possui baixo poder neutralizante e não aumentou o pH, logo não deve ser aplicado ao solo como adubo ou corretivo agrícola.

Entre os parâmetros que limitam a disposição do lodo no solo está o mangânes que é o metal em maior concentração e restringiu a aplicação a uma taxa de $2,5 \mathrm{~kg} \mathrm{~m}^{-2}$. Outro elemento restritivo é o $\mathrm{Fe}$, porém os teores naturalmente elevados de $\mathrm{Fe}$ no solo analisado podem ter comprometido a avaliação para esse metal.

Apesar do lodo de ETA não ter elevado as bases a valores significativos, o lodo foi capaz de reduzir em $20 \%$ o índice de saturação por alumínio através da adição dos nutrientes $\mathrm{Ca}$ e $\mathrm{Mg}$ na maior dose aplicada. Contudo, a restrição devido ao manganês (a uma taxa de aplicação de 2,5 $\mathrm{kg} \mathrm{m}^{-2}$ ) implicou que a redução do índice de saturação por alumínio seria de no máximo 8\%.

Há indícios que a aplicação de lodo de ETA no solo pode ter como finalidade a recuperação de áreas degradadas uma vez que o lodo não provocou a salinização maior dose aplicada e atuou como agente cimentante e coagulante das partículas tornando o solo mais poroso o que favorece a penetração das raízes, a aeração, a taxa de infiltração, a difusão do oxigênio, a atividade biológica, melhorando assim a estrutura do solo e o desenvolvimento das plantas.

\section{REFERÊNCIAS}

ADLER, E. Management of wastes from drinking water treatment. In: INTERNATIONAL CONFERENCE, ORGANISED BY THE CHARTERED INSTITUTION OF WATER AND ENVIRONMENTAL MANAGEMENT. London, 2002.

AMERICAN WATER WORKS ASSOCIATION RESEARCH FOUDATION AWWARF. Commercial Application and Marketing of Water Plant Residuals. USA 1999. 
AGYIN-BIRIKORANG, S.; O'CONNOR, G. A.; OBREZA, T. Are Alum-Based Drinking Water Treatment Residuals Safe for Land Application? INSTITUTE OF FOOD AND AGRICULTURAL SCIENCES - IFAS. University of Florida. Florida, 2009.

ANDREOLI, C. V (coord.). Usos alternativos de lodos de estação de tratamento de água e estação de tratamento de esgoto. 1a ed. Rio de Janeiro: ABES, 2006. 417 p. Programa de Pesquisa em Saneamento Básico - PROSAB.

APHA; AWWA; WEF. Standard methods for the examination of water andwastewater. $21^{\circ}$ ed. Washington: APHA, 2005.

BASSO, C. J. CERETTA, C. A.; FLORES, E. M. M.; GIROTTO, E. Teores totais de metais pesados no solo após aplicação de dejeto líquido de suínos. Ciência Rural. v.42, n.4, p.653-659, 2012.

BITTENCOURT, S.; SERRAT, M. B.; AISSE, M. M.; MARIN, L. M. K. S.; SIMÃO, C. C. Aplicação de lodos de estações de tratamento de água e de tratamento de esgoto em solo degradado. Engenharia Sanitária e Ambiental, v. 17, n. 3, p. 315-324, 20102.

BRADY, N. C.; WEIL, R. R. Elementos da natureza e propriedades dos solos. Editora Bookmam Companhia LTDA. $3^{\circ}$ edição. Porto Alegre, 2013. 655p.

BRASIL. Conselho Nacional de Meio Ambiente - CONAMA. Resolução $n^{\circ} 420$, de 28 de dezembro de 2009. Dispõe sobre critérios e valores orientadores de qualidade do solo quanto à presença de substâncias químicas e estabelece diretrizes para o gerenciamento ambiental de áreas contaminadas por essas substâncias em decorrência de atividades antrópicas. Publicado no Diário Oficial da União em 30.12.2009.

EMPRESA BRASILEIRA DE PESQUISA AGROPECUÁRIA - EMBRAPA. Manual de métodos de análise de solo. $2^{\circ}$ edição Revista. Rio de Janeiro, 2011. 225p.

FERREIRA, D. F. Sisvar: a computer statistical analysis system. Ciência e Agrotecnologia. v. 35, n.6, p. 1039-1042, 2011.

INSTITUTO BRASILEIRO DE GEOGRAFIA E ESTATÍSTICA. Pesquisa Nacional de Saneamento Básico 2008 (PNSB). Rio de Janeiro. 2010.

INSTITUTO NACIONAL DE METEOROLOGIA - INMET. Disponível em: <http://www.inmet.gov.br/portal/index.php?r=bdmep/bdmep>. Acesso em: 27 de janeiro de 2015.

IPPOLITO, J. A.; BARBARICK, K. A.; ELLIOTT, H. A. Drinking Water Treatment Residuals: A Review of Recent Uses. Journal of Environmental Quality. 40, p.1-12, 2011. 
KER, J. C. Latossolos do Brasil: uma revisão. Geonomos, v. 15, n. 1, p. 17- 40, 1991.

KROB, A. D.; MORAES, S. P.; SELBACH, P. A.; BENTO, M. F.; CAMARGO, F. A. O. Propriedades químicas de um argissolo tratado sucessivamente com composto de lixo urbano. Ciência Rural. v.41, n.3, p.433-439, 2011.

MAHDY, A.M.; ELKHATIB, E. A.; LIN, Z. Q. Effects of drinking water treatment residuals on soil solution composition and phosphorus speciation in biosolidamended soils of Kafr El-Dawar, Egypt, and Troy, USA. Agrochimica, v.3, n. 4, p.315-336, 2013.

MALAVOLTA, E. Elementos de nutrição mineral de plantas. Editora Agrônomica CEREs, 1980. 251p.

MAPA DE SOLOS DO ESTADO DE MINAS GERAIS: legenda expandida. Universidade Federal de Viçosa; Fundação Centro Tecnológico de Minas Gerais; Universidade Federal de Lavras; Fundação Estadual do Meio Ambiente. Belo Horizonte: Fundação Estadual do Meio Ambiente, 2010.49p.

MATOS, A. T. Tratamento e aproveitamento agrícola de resíduos sólidos. Associação dos Engenheiros Agrícolas de Minas Gerais, 2007, 120p.

MATOS, A. T. Tratamento e aproveitamento agrícola de resíduos sólidos. Viçosa, Ed. UFV, 2014, 241p.

MEGDA, C. R.; SOARES, L. V.; ACHON, C. L.; Proposta de Aproveitamento de Lodos Gerados em ETAs. In: XXIII CONGRESSO BRASILEIRO DE ENGENHARIA SANITÁRIA E AMBIENTAL. Campo Grande.Anais, 2005.

MERLINO, L. C. S. et al. Bário, cádmio, cromo e chumbo em plantas de milho e em latossolo após onze aplicações anuais de lodo de esgoto. Revista Brasileira Ciência do Solo. v.34, n.6, 2010.

MOREIRA, R. S. Aplicação de lodo de esgoto em latossolo distrófico: fertilidade e disponibilidade de metais pesados. Dissertação (Tecnologia Ambiental). Universidade Federal de Alfenas, 2013.

PADILHA, J. C. Aplicação de lodos de tratamentos de água e esgoto em latossolos cultivados com milho e soja. Dissertação (Ciência do Solo). Universidade Federal do Paraná. Curitiba, 2007. 346f.

PEREIRA, S. L. M. Características físicas, químicas e microbiológicas do lodo das lagoas da ETA Gramame. Dissertação (Mestrado em Engenharia Urbana e Ambiental) - Centro de Tecnologia, Universidade Federal da Paraíba, João Pessoa, 2011.

RIBEIRO, A.C.; GUIMARÃES, P.T.G.; ALVAREZ V., V.H. Recomendação para 0 uso de corretivos e fertilizantes em Minas Gerais. $5^{\circ}$ aproximação. Viçosa, MG: Comissão de Fertilidade do Solo do Estado de Minas Gerais, 1999a. 359p. 
RIBEIRO, A.C.; GUIMARÃES, P.T.G.; ALVAREZ V., V.H. Recomendação para 0 uso de corretivos e fertilizantes em Minas Gerais - Pastagens. $5^{\circ}$ aproximação. Viçosa, MG: Comissão de Fertilidade do Solo do Estado de Minas Gerais, 1999b. 7p.

RONQUIM, C. C. Conceitos de fertilidade do solo e manejo adequado para as regiões tropicais. Empresa Brasileira de Pesquisa Agropecuária - EMBRAPA, Embrapa Monitoramento por Satélite e Ministério da Agricultura, Pecuária e Abastecimento. ISSN 1806-3322. 2010.

SANTOS, R. D.; LEMOS, R. C.; SANTOS, H. G.; KER, J. C.; ANJOS, L. H. C. SHIMIZU, S. H. Manual de descrição e coleta de solo no campo. $6^{\circ}$ ed. Sociedade Brasileira de Ciência do Solo. 2013. 100p.

SOCIEDADE BRASILEIRA DE CIÊNCIA DO SOLO - SBCS. Manual de adubação e de calagem para os estados do rio grande do sul e de Santa Catarina. Comissão de Química e Fertilidade do Solo. 2004

TEIXEIRA, S. T.; MELO, W. J.; SILVA, E. T. Aplicação de lodo da estação de tratamento de água em solo degradado. Pesquisa Agropecuária Brasileira. Brasília, v.40, n.1, p.91-94. 2005.

TUCCI, C.E.M. (organizador). Hidrologia: Ciência e Aplicação. Ed. Da Universidade - UFRGS, Ed. da Universidade de São Paulo - EDUSP e Associação Brasileira de Recursos Hídricos - ABRH, 1ª edição. 1993.

Recebido:04/11/2015

Aprovado:10/12/2015

Revista Internacional de Ciências · v.5 - n.2 · jul./dez. 2015 